\title{
The One-Filter Keefe Clarinet Tonehole
}

\author{
Julius O. Smith III, Gary P. Scavone \\ Center for Computer Research in Music and Acoustics (CCRMA) \\ (http://www-ccrma.stanford.edu/) \\ Stanford University, Stanford, CA 94305 USA \\ jos@ccrma.stanford.edu,gary@ccrma.stanford.edu
}

\begin{abstract}
Two "one-filter" scattering junctions are derived which provide very accurate models of woodwind toneholes in the context of a digital waveguide model. Because toneholes in the clarinet possess only one resonance and/or anti-resonance within the audio band, a second-order digital filter suffices.
\end{abstract}

\section{Introduction}

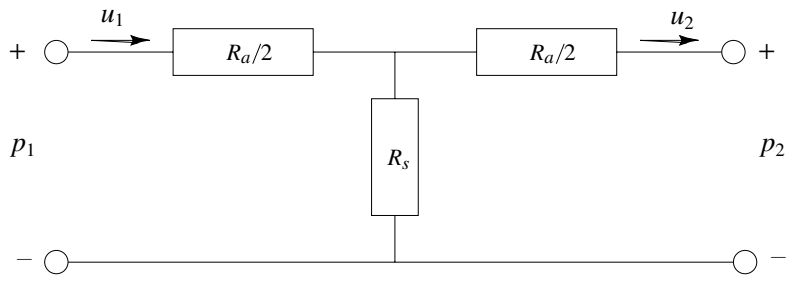

Figure 1: Lumped-parameter description of the clarinet tonehole.

The clarinet tonehole model developed by Keefe [1] is parametrized in terms of series and shunt resistance and reactance, as shown in Fig. 1. The transmission-matrix description of this two-port is given by the product of the transmission matrices for the series impedance $R_{a} / 2$, shunt impedance $R_{s}$, and series impedance $R_{a} / 2$, respectively:

$$
\begin{aligned}
{\left[\begin{array}{l}
P_{1} \\
U_{1}
\end{array}\right] } & =\left[\begin{array}{cc}
1 & R_{a} / 2 \\
0 & 1
\end{array}\right]\left[\begin{array}{cc}
1 & 0 \\
R_{s}^{-1} & 1
\end{array}\right]\left[\begin{array}{cc}
1 & R_{a} / 2 \\
0 & 1
\end{array}\right]\left[\begin{array}{c}
P_{2} \\
U_{2}
\end{array}\right] \\
& =\left[\begin{array}{cc}
1+\frac{R_{a}}{2 R_{s}} & R_{a}\left[1+\frac{R_{a}}{4 R_{s}}\right] \\
\frac{1}{R_{s}} & 1+\frac{R_{a}}{2 R_{s}}
\end{array}\right]\left[\begin{array}{c}
P_{2} \\
U_{2}
\end{array}\right]
\end{aligned}
$$

where all quantities are written in the frequency domain, and the impedance parameters are given by

$$
\begin{aligned}
\text { (open-hole shunt imp.) } & R_{s}^{o}=R_{b}\left(j k t_{e}+\xi_{e}\right) \\
\text { (closed-hole shunt imp.) } & R_{s}^{c}=-j R_{b} \cot \left(k t_{h}\right) \\
\text { (open-hole series imp.) } & R_{a}^{o}=-j R_{b} k t_{a}^{o} \\
\text { (closed-hole series imp.) } & R_{a}^{c}=-j R_{b} k t_{a}^{c}
\end{aligned}
$$

where $R_{b}=\rho c /\left(\pi b^{2}\right)$ is the wave impedance of the tonehole entrance, i.e., that of an acoustic tube of crosssectional area $\pi b^{2}$ ( $\rho$ is air density and $c$ is sound speed as usual), $b$ is the tonehole radius, $k=\omega / c=2 \pi / \lambda$ is the wavenumber (radian spatial frequency), $t_{e}$ is the open-tonehole effective length, $\xi_{e}$ is the "specific resistance" of the open tonehole due to air viscosity in and radiation from the hole, $t_{h}$ is the closed-tonehole height. Finally, $t_{a}^{o}$ and $t_{a}^{c}$ are the equivalent series lengths of the open and closed tonehole, respectively, described further in $[1]$.

For implementation in a digital waveguide model [2], the lumped parameters above must be converted to scattering parameters. Such formulations of toneholes have recently appeared [3, 4]. In [5], a four-filter model was developed based on the "symmetric T" model of Keefe above. A detailed treatment appears in [6]. This paper derives two stable one-filter forms which also include precisely the effects of the series reactance in Keefe's model.

Substituting $k=\omega / c$ in (2) to convert spatial frequency to temporal frequency, and substituting

$$
\begin{aligned}
P_{i} & =P_{i}^{+}+P_{i}^{-} \\
U_{i} & =\frac{P_{i}^{+}-P_{i}^{-}}{R_{0}}
\end{aligned}
$$

for $i=1,2$, into (1) to convert physical variables to wave variables, $\left(R_{0}=\rho c /\left(\pi a^{2}\right)\right.$ is the bore wave-impedance), and solving for the outgoing waves $P_{1}^{-}, P_{2}^{-}$in terms of the incoming waves $P_{1}^{+}, P_{2}^{+}$, we obtain

$$
\left[\begin{array}{c}
P_{1}^{-} \\
P_{2}^{+}
\end{array}\right]=\left[\begin{array}{cc}
S & T \\
T & S
\end{array}\right]\left[\begin{array}{c}
P_{1}^{+} \\
P_{2}^{-}
\end{array}\right]
$$

where

$$
S(\omega)=\frac{4 R_{a} R_{s}+R_{a}^{2}-4 R_{0}^{2}}{\left(2 R_{0}+R_{a}\right)\left(2 R_{0}+R_{a}+4 R_{s}\right)} \approx-\frac{R_{0}}{R_{0}+2 R_{s}}
$$

is the reflectance of the tonehole (the same from either direction), and

$$
T(\omega)=\frac{8 R_{0} R_{s}}{\left(2 R_{0}+R_{a}\right)\left(2 R_{0}+R_{a}+4 R_{s}\right)} \approx \frac{2 R_{s}}{R_{0}+2 R_{s}}
$$

is the transmittance of the tonehole (also the same from either direction). The resulting scattering formulation 
is depicted in Fig. 2. The notation " $S$ " for reflectance is chosen because every reflectance is a Schur function (stable and not exceeding unit magnitude on the unit circle in the $z$ plane).

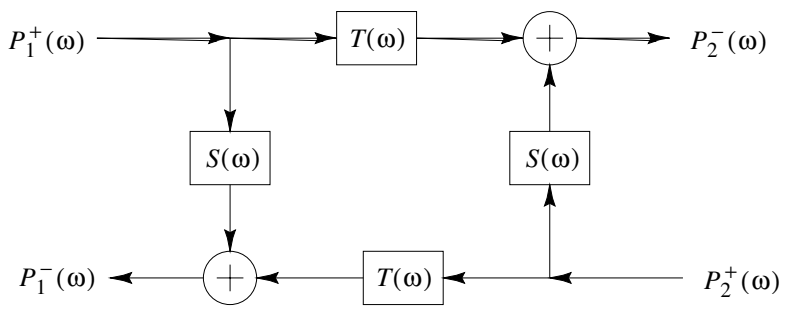

Figure 2: Frequency-domain, traveling-wave description of the clarinet tonehole.

The approximate forms in (6) and (7) are obtained by neglecting the negative series inertance $R_{a}$ which serves to adjust the effective length of the bore, and which therefore can be implemented outside of the tonehole as derived below. The open and closed tonehole cases are obtained by substituting $\left\{R_{a}=R_{a}^{o}, R_{s}=R_{s}^{o}\right\}$ and $\left\{R_{a}=R_{a}^{c}, R_{s}=R_{s}^{c}\right\}$, respectively, from (2).

In a manner analogous to converting the four-multiply Kelly-Lochbaum (KL) scattering junction into a onemultiply form [7] we may pursue a "one-filter" form of the waveguide tonehole model. However, the series inertance gives some initial trouble, since

$$
[1+S(\omega)]-T(\omega)=\frac{2 R_{a}}{2 R_{0}+R_{a}} \triangleq L(\omega)
$$

instead of zero as in the KL junction. In the general scattering formulas for an $N$-way waveguide junction loaded by an arbitrary lumped impedance [8], the reflectance seen on any branch is always the transmittance from that branch to any other branch minus 1. I.e., if $\alpha_{i}$ denotes the transmittance from branch $i$ to all other branches meeting at the junction, then $\alpha_{i}-1$ is the reflectance seen on branch $i$. Substituting

$$
T=1+S-L
$$

into the scattering relations (5), and factoring out $S$, we obtain, in the frequency domain,

$$
\begin{aligned}
P_{1}^{-}(\omega) & =S P_{1}^{+}+T P_{2}^{+} \\
& =S P_{1}^{+}+[1+S-L] P_{2}^{+} \\
& =S\left[P_{1}^{+}+P_{2}^{+}\right]+[1-L] P_{2}^{+} \\
& \triangleq S\left[P_{1}^{+}+P_{2}^{+}\right]+A P_{2}^{+}
\end{aligned}
$$

and, similarly,

$$
P_{2}^{-}(\omega)=S\left[P_{1}^{+}+P_{2}^{+}\right]+A P_{1}^{+}
$$

The resulting tonehole implementation is shown in Fig. 3. We call this the "shared reflectance" form of the tonehole junction.

In the same way, an alternate form is obtained from the substitution

$$
S=T-1+L
$$

which yields the "shared transmittance" form:

$$
\begin{aligned}
& P_{1}^{-}=T\left[P_{1}^{+}+P_{2}^{+}\right]-A P_{1}^{+} \\
& P_{2}^{-}=T\left[P_{1}^{+}+P_{2}^{+}\right]-A P_{2}^{+}
\end{aligned}
$$

shown in Fig. 4.

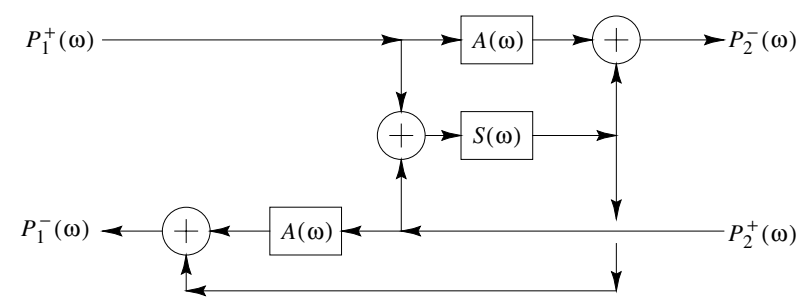

Figure 3: "Shared-reflectance" implementation of the clarinet tonehole model.

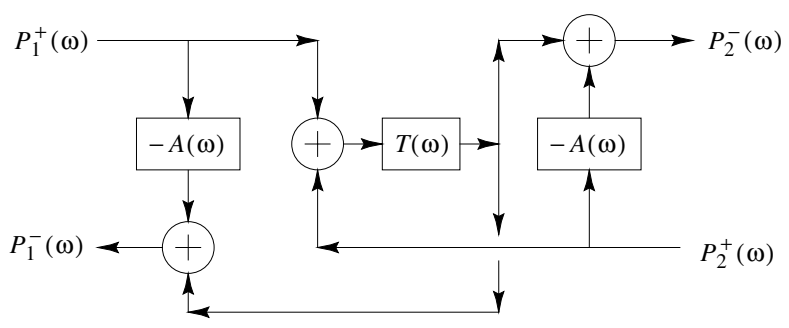

Figure 4: "Shared-transmittance" implementation of the clarinet tonehole model.

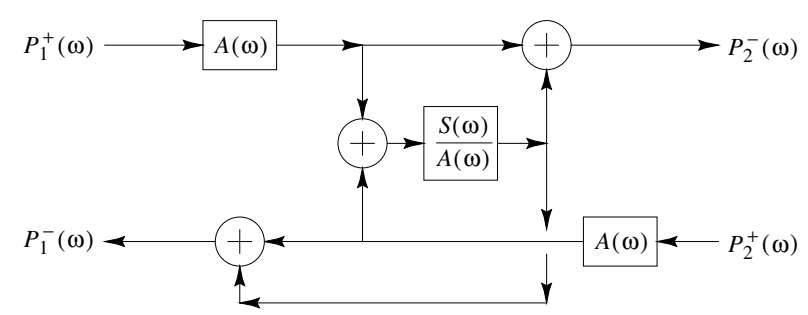

Figure 5: "Shared-reflectance" tonehole model with unstable allpasses pulled out to the inputs. 


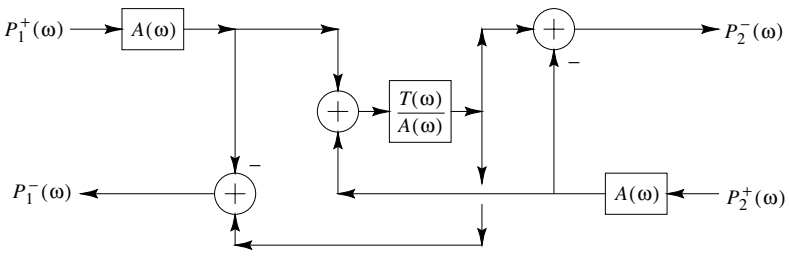

Figure 6: "Shared-transmittance" tonehole model with unstable allpasses pulled out to inputs.

Since $L(\omega) \approx 0$, it can be neglected to first order, and $A(\omega) \approx 1$, reducing both of the above forms to an approximate "one-filter" tonehole implementation.

Since $R_{a}=-j R_{b} \omega t_{a} / c$ is a pure negative reactance, we have

$$
A(\omega)=1-L(\omega)=\frac{R_{0}-R_{a} / 2}{R_{0}+R_{a} / 2}=\frac{p+j \omega}{p-j \omega}, \quad p=\frac{R_{0} c}{R_{b} t_{a}}
$$

In this form, it is clear that $A(\omega)$ is a first-order allpass filter with a single pole-zero pair near infinity. Unfortunately, the pole is in the right-half-plane and hence unstable. We cannot therefore implement it as shown in Fig. 3 or Fig. 4. Using elementary manipulations, the unstable allpasses in Figs. 3 and Fig. 4 can be moved to the configurations shown in Figs. 5 and 6 , respectively. Note that $T(\omega) / A(\omega)$ is stable whenever $T$ is stable, and $T$ is properly constrained to be stable because it is a passive transmittance. The unstable allpasses now operate only on the two incoming wave variables, and they can be implemented implicitly by slightly reducing the (interpolated) delay-lines leading to the junction from either side. The tonehole then requires only one filter $S / A$ or $T / A$.

We now see precisely how the negative series inertance $R_{a}$ provides a negative, frequency-dependent, length correction for the bore. From the preceding equation, the phase delay of $A(\omega)$ is

$D_{A}(\omega) \triangleq-\frac{L A(\omega)}{\omega}=-2 \tan ^{-1}\left(\frac{\omega}{p}\right)=-2 \tan ^{-1}\left(\frac{k t_{a} R_{b}}{R_{0}}\right)$

Thus, the negative delay correction goes to zero with frequency $k=\omega / c$, series tonehole length $t_{a}$, tonehole impedance $R_{b}$, or main bore admittance $\Gamma_{0}=1 / R_{0}$.

In practical digital waveguide modeling, it is common to combine all delay corrections into a single "tuning allpass filter" for the whole bore $[9,10]$. Whenever the desired allpass delay goes negative, we simply add a sample of delay to the desired allpass phase-delay and subtract it from the nearest delay line. In other words, negative delays have to be "pulled out" of the allpass and used to shorten an adjacent interpolated delay line. Since the tonehole connects on either side to a section of bore, and since the digital waveguide model for a bore includes a delay line, adjacent delay lines for negative-delay absorption are normally available in practical modeling situations.

\subsection{Tonehole Filter Design}

The tone-hole reflectance and transmittance must be converted to discrete-time form for implementation in a digital waveguide model. Figure 7 plots the responses of second-order discrete-time filters designed to approximate the continuous-time magnitude and phase characteristics of the modified reflectance $S(\omega) / A(\omega)$ for closed and open toneholes, as carried out in $[5,6]$. These filter designs assumed a tonehole of radius $b=4.765 \mathrm{~mm}$, minimum tonehole height $t_{w}=3.4 \mathrm{~mm}$, tonehole radius of curvature $r_{c}=0.5 \mathrm{~mm}$, and air column radius $a=9.45$ $\mathrm{mm}$. Because the responses provided by Keefe were experimentally verified only for frequencies less than $5 \mathrm{kHz}$, the filter designs were weighted to produce best results below this limit.

The closed-hole filter design was carried out using weighted $L_{2}$ equation-error minimization [9, p. 47], i.e., by minimizing $\left\|W\left(e^{j \Omega}\right)\left[\hat{A}\left(e^{j \Omega}\right) H\left(e^{j \Omega}\right)-\hat{B}\left(e^{j \Omega}\right)\right]\right\|_{2}$, where $W$ is the weighting function, $H\left(e^{j \Omega}\right)$ is the desired frequency response, $\Omega$ denotes discrete-time radian frequency, and the designed filter response is $\hat{H}\left(e^{j \Omega}\right)=$ $\hat{B}\left(e^{j \Omega}\right) / \hat{A}\left(e^{j \Omega}\right)$. Note that both phase and magnitude are matched by equation-error minimization, and this error criterion is used extensively in the field of system identification due to its ability to design optimal IIR filters via quadratic minimization. In the spirit of the wellknown Steiglitz-McBride algorithm, equation-error minimization can be iterated, setting the weighting function at iteration $i+1$ to the inverse of the inherent weighting $\hat{A}_{i}$ of the previous iteration, i.e., $W_{i+1}\left(e^{j \Omega}\right)=1 / \hat{A}_{i}\left(e^{j \Omega}\right)$. However, for this study, the weighting was used only to increase accuracy at low frequencies relative to high frequencies. Weighted equation-error minimization is implemented in the Matlab function invfreqz().

The open-hole discrete-time filter was designed using Kopec's method [7], [9, p. 46] in conjunction with weighted equation-error minimization. Kopec's method is based on linear prediction:

1. Given a desired complex frequency response $H\left(e^{j \Omega}\right)$, compute an allpole model $1 / \hat{A}(z)$ using linear prediction, i.e., by minimizing the $L_{2}$ ratio error $\left\|\hat{A}\left(e^{j \Omega}\right) H\left(e^{j \Omega}\right)\right\|_{2}$. 
2. Compute the error spectrum $\hat{E} \triangleq \hat{A} H$.

3. Compute an allpole model $1 / \hat{B}(z)$ for $\hat{E}^{-1}\left(e^{j \Omega}\right)$ by minimizing

$$
\left\|\hat{B}\left(e^{j \Omega}\right) \hat{E}^{-1}\left(e^{j \Omega}\right)\right\|_{2}=\left\|\frac{\hat{B}\left(e^{j \Omega}\right)}{\hat{A}\left(e^{j \Omega}\right)} H^{-1}\left(e^{j \Omega}\right)\right\|_{2} .
$$

The ratio error used in linear prediction causes the filter to fit the upper spectral envelope of the desired frequencyresponse. Since the first step of Kopec's method captures the upper spectral envelope, the "nulls" and "valleys" are largely "saved" for the next step which computes zeros. When computing the zeros, the spectral "dips" become "peaks," thereby receiving more weight under the $L_{2}$ ratio-error norm. Thus, in Kopec's method, the poles model the upper spectral envelope, while the zeros model the lower spectral envelope. To apply Kopec's method to the design of an open-tonehole filter, a onepole model $\hat{H}_{1}(z)$ was first fit to the continuous-time response, $H\left(e^{j \Omega}\right)$. Subsequently, the inverse error spectrum, $\hat{H}_{1}\left(e^{j \Omega}\right) / H\left(e^{j \Omega}\right)$ was modeled with a two-pole digital filter, $\hat{H}_{2}(z)$. The discrete-time approximation to $H\left(e^{j \Omega}\right)$ was then given by $\hat{H}_{1}(z) / \hat{H}_{2}(z)$, as shown in Fig. 7 .
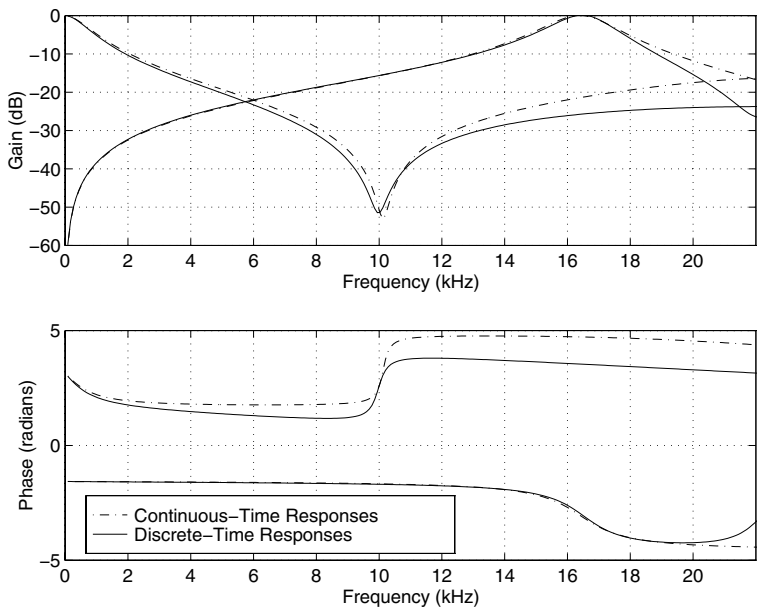

Figure 7: Two-port tonehole junction closed-hole and open-hole reflectances based on Keefe's acoustic measurements (dashed) versus second-order digital filter approximations (solid). Top: Reflectance magnitude; Bottom: Reflectance phase. The closed tonehole has one resonance in the audio band just above $16 \mathrm{kHz}$. The open tonehole has one anti-resonance in the audio band near $10 \mathrm{kHz}$. At DC, the open tonehole fully reflects, while the closed tonehole is close to non-reflecting.

The reasonably close match in both phase and magnitude by second-order filters indicates that there is in fact only one important tonehole resonance and/or antiresonance within the audio band, and that the measured frequency responses can be modeled with very high audio accuracy using only second-order filters.

\section{Conclusions}

A second-order digital reflectance/transmittance model was based on the experimentally confirmed acoustic model for the clarinet tonehole by Keefe. It was therefore shown that precise models of tonehole acoustics can be incorporated into digital waveguide synthesis models of woodwind musical instruments for relatively little added computational cost. Linear tonehole acoustic features are handled very well by a second-order digital tonehole filter. However, nonlinear acoustic effects, such as vortex shedding and flow separation, are not addressed in this linear model, and provide subjects for further research.

\section{References}

1. D. H. Keefe, "Woodwind air column models", J. Acoustical Soc. of America, vol. 88, no. 1, pp. 35-51, Jan. 1990.

2. J. O. Smith, "Physical modeling synthesis update", Computer Music J., vol. 20, no. 2, pp. 44-56, Summer 1996, Available online at http://www-ccrma.stanford.edu/ jos/.

3. V. Välimäki, M. Karjalainen, and T. I. Laakso, "Modeling of woodwind bores with finger holes", in Proc. 1993 Int. Computer Music Conf., Tokyo. 1993, pp. 3239, Computer Music Assoc.

4. V. Välimäki, Discrete-Time Modeling of Acoustic Tubes Using Fractional Delay Filters, PhD thesis, Report no. 37, Helsinki University of Technology, Faculty of Elec. Eng., Lab. of Acoust. and Audio Sig. Proc., Espoo, Finland, Dec. 1995.

5. G. Scavone and J. O. Smith, "Digital waveguide modeling of woodwind toneholes", in Proc. 1997 Int. Computer Music Conf., Greece. 1997, Computer Music Assoc.

6. G. P. Scavone, An Acoustic Analysis of Single-Reed Woodwind Instruments with an Emphasis on Design and Performance Issues and Digital Waveguide Modeling Techniques, $\mathrm{PhD}$ thesis, Music Dept., Stanford University, March 1997, CCRMA Tech. Rep. STANM-100. Available also via ftp://ccrmaftp.stanford.edu/pub/Publications/Theses/GaryScavoneThesis/.

7. J. D. Markel and A. H. Gray, Linear Prediction of Speech, Springer Verlag, New York, 1976.

8. J. O. Smith, "Music applications of digital waveguides", Tech. Rep. STAN-M-39, CCRMA, Music Dept., Stanford University, 1987, (Order via email to info@ccrma.stanford.edu).

9. J. O. Smith, Techniques for Digital Filter Design and System Identification with Application to the Violin, PhD thesis, Elec. Eng. Dept., Stanford, June 1983.

10. D. A. Jaffe and J. O. Smith, "Extensions of the KarplusStrong plucked string algorithm", Computer Music J., vol. 7, no. 2, pp. 56-69, 1983, Reprinted in C. Roads, Ed., The Music Machine, MIT Press, Cambridge, MA, 1989. 